\title{
Sinonasal Adenoid Cystic Carcinoma: A Case Report and Review of the Literature
}

\author{
*Zakaria Chafiki, Ait El kerdoudi M, Merzouki B, Rouadi S, Abada R, Roubal M and Mahtar M \\ ENT-Head and Neck surgery, Ibn Rochd University Hospital, Morocco
}

Submission: November 28, 2016; Published: December 06, 2016

"Corresponding author: CHAFIKI Zakaria, ENT-Head and Neck surgery, August $20^{\text {th }}$ Hospital, Ibn Rochd University Hospital, Casablanca, Morocco.

\begin{abstract}
Adenoid cystic carcinomas (ACC) represent $10 \%$ of all salivary tumors. It primarily affects the salivary glands particularly those located in the buccal cavity. Sinonasal location is rarely described [1]. We report a rare case of sinonasal adenoid cystic carcinoma (SACC), we discuss through a brief review of the literature its clinical, radiological, histopathological and therapeutic modalities and the prognostic factors of this tumor.
\end{abstract}

Keywords: Adenoid Cystic Carcinoma; Paranasal Sinus; Facial Pain

\section{Introduction}

ACC is a rare malignant neoplasm that accounts for $1-2 \%$ of all head and neck malignancies and approximately $10 \%$ of all salivary gland neoplasms [2]. It occurs predominantly among women, between the fifth and sixth decades of life [3]. Sinonasal Adenocarcinomas mainly arise from the respiratory epithelium or the underlying mucoserous glands (60\%). It's known by its slow persistent growth with a high risk of local recurrences and distant metastases. The treatment of choice is based on surgical excision of the tumor with adjuvant radiation therapy.

\section{Case Report}

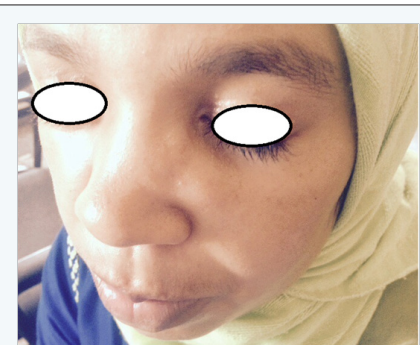

Figure 1: Swelling of the latero-nasal area and the inner corner of the left eye.

A 44 year-old female presented to the ENT emergencies with a swelling of the latero-nasal area and the inner corner of the left eye evolving for the last year. It measured $2.5 \mathrm{~cm}$ at its largest diameter (Figure 1). The patient reported a progressive left-sided nasal obstruction of 8 months duration, with purulent,bloody and fetid nasal discharge, with a conservation of the general state. The anterior rhinoscopy highlighted nasal congestion with no visible tumor. Ophthalmologic examination revealed lateralization of the left eyeball with telecanthus. Facial CT scan revealed a tumor process of the left nasolabial furrow with lysis of the maxillary bone, its internal wall and also the inner wall of the orbit (Figure 2). A biopsy through the vestibular way has confirmed the diagnosis of a cribriformtype of ACC. The staging did not find distant metastases. The patient was managed surgically with adjuvant radiation therapy.

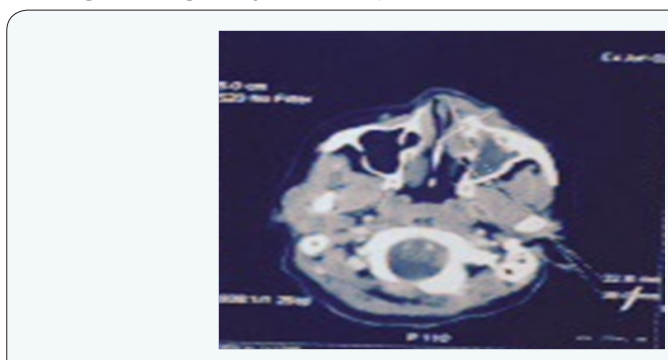

Figure 2: CT scan-Axial section-(maxillary sinus) tumor mass of the left nasolabial fold with lysis of the maxillary bone, internal wall and also the inner wall of the orbit.

\section{Discussion}

Sinonasal Adenoid cystic carcinomas are considered as poor prognostic tumors, characterized by the possibility of late and frequent local recurrences, and poor survival. These tumors grow usually slowly, thus they can reach considerable size before the diagnosis is made. The initial prognosis may be good but the 


\section{Cancer Therapy \& Oncology International Journal}

frequency of local recurrences and distant metastases (lung essentially) influence the long-term survival (10-year survival $<10 \%$ ) [4]. Local recurrences are more frequent for sinonasal locations $(60 \%$ of clinically evident recurrences within 2 years following treatment) [5]. This is related to the difficulty of ensuring healthy resection margins at the base of the skull often because of the very advanced stage of the tumor by the time of the diagnosis, the anatomic complexity of the region, the frequent intracranial extension along cranial nerves and because of restriction on the resection margins caused by the proximity of critical neurovascular structures.

Among the most important prognostic factors involved are tumor stage, histological grade (The tubular- and cribriformtype ACCs are lower-grade tumors, whereas solid-type ACC is a higher-grade tumor), the existence of perineural invasion and cancerous resection margins $[4,6,7]$. Complete surgical resection is critical but often difficult to realize close to the skull base $[4,8]$. Postoperative radiotherapy improves the long-term prognosis of patients with large lesions especially if there are microscopic residues after surgery.

\section{Conclusion}

Adenoid cystic carcinoma of sinonasal cavities is a rare aggressive tumor with a high incidence of local recurrence and distance metastases regardless of therapeutic modalities used. Complete surgical excision and adjuvant radiation therapy for the extensive local infiltration offer to these patients the best chance to achieve high tumor control.

\section{References}

1. Bradley PJ (2004) Adenoid cystic carcinoma of the head and neck: a review. Curr Opin Otolaryngol Head Neck Surg 12: 127-132.

2. Falk GA, El-Hayek K, Morris-Stiff G, Tuthill RJ, Winans CG (2011) Adenoid cystic carcinoma of the base of the tongue: Late metastasis to the pancreas. Int J Surg Case Rep 2: 1-3.

3. Safinaz Zainor, Hamidah Mamat, Sakina Mohd Saad, Mohd Razif Mohamad Yunus (2013) Adenoid cystic carcinoma of external auditory canal: A case report. Egyptian Society of Ear, Nose, Throat and Allied Sciences 14(1): 41-44.

4. Konno A, Ishikawa K, Numata T, Nagata H, Terada N, Okamoto Y (1998) Analysis of factors affecting long-term treatment results of adenoid cystic carcinoma of the nose and paranasal Sinuses. Acta Otolaryngol (Stockh) Suppl 537: 67-74.

5. Wiseman SM, Popat SR, Rigual NR, Hicks WL Jr, Orner JB, et al. Adenoid cystic carcinoma of the paranasal sinuses or nasal cavity: a 40-year review of 35 cases. Ear Nose Throat J 81(8): 510-514, 516-517.

6. Naficy S, Disher MJ, Esclamado RM (1999) Adenoid cystic carcinoma of the paranasal sinuses. Am J Rhinol. 13(4): 311-314.

7. Sato K, Ueda Y, Sakurai A, Ishikawa Y, Kaji S, et al. (2006) Adenoid cystic carcinoma of the maxillary sinus with gradual histologic transformation to high-grade adenocarcinoma: A comparative report with dedifferentiated carcinoma. Virchows Arch 448(2): 204-208.

8. Prott FJ, Micke O, Haverkamp U, Willich N, Schuller P, et al. (2000) Results of fast neutron therapy of adenoid cystic carcinoma of the salivary glands. Anticancer Res 20(5C): 3743-3750. 